\title{
Crecimiento de plántulas de cacao (Theobroma cacao L.) en diferentes tamaños de contenedor
}

\author{
Cacao (Theobroma cacao L.) seedlings growth in different pot sizes
}

\author{
Mónica Andrea Osorio G. ${ }^{1}$; Edna Ivonne Leiva R ${ }^{2}$; Ramiro Ramírez P. ${ }^{3}$
}

\footnotetext{
${ }^{1}$ I.A. Facultad de Ciencias Agrarias, Universidad Nacional de Colombia Sede Medellín, Medellín, Colombia, maosorio@unal.edu.co.

${ }^{2}$ Profesora Asociada. Ph.D. Facultad de Ciencias Agrarias, Universidad Nacional de Colombia Sede Medellín, Medellín, Colombia, eileiva@unal.edu.co.

${ }^{3}$ Profesor Asociado. Ph.D. Facultad de Ciencias, Universidad Nacional de Colombia Sede Medellín, Medellín, Colombia, rramirez@unal.edu.co.
}

Citar: Osorio, M.; Leiva, E.; Ramírez, R. 2017. Crecimiento de plántulas de cacao (Theobroma cacao L.) en diferentes tamaños de contenedor. Rev. Cienc. Agr. 34(2): 73 - 82. doi: http://dx.doi.org/10.22267/rcia.173402.73.

Recibido: Septiembre 08 de $2016 . \quad$ Aceptado: Mayo 02 de 2017.

\section{RESUMEN}

El volumen del contenedor para la propagación del cacao es determinante, ya que su tamaño influye en el crecimiento y desarrollo de las plántulas, siendo un factor definitivo para el establecimiento del cultivo. En este trabajo se comparó el efecto que tienen diferentes tamaños de contenedor 0,$01 ; 0,02$ y $0,1 \mathrm{~m}^{3}$ (volumen de sustrato) y la duración en vivero, sobre el crecimiento de plantas de cacao, además, se evaluaron los cambios en el sustrato confinado. Se sembraron semillas de cacao en contenedores de diferentes alturas: 25, 45 y $70 \mathrm{~cm}$. Se midió la temperatura de las hojas y se colectaron plántulas, para cuantificar la biomasa particionada y crecimiento de la raíz principal. Simultáneamente fueron evaluadas la resistencia a la penetración, la temperatura, la humedad y la porosidad del sustrato. Los resultados evidenciaron diferencias significativas $(\mathrm{P}<0,05)$ en la acumulación de fitomasa total. En los contenedores de mayor tamaño $\left(0,1 \mathrm{~m}^{3}\right)$ hubo mayor biomasa total, mayor longitud de raíces: $62,17 \mathrm{~cm}$, el sustrato presentó menor resistencia a la penetración $\left(0,52 \mathrm{Kg} \mathrm{cm}^{-2}\right)$ y temperatura. La temperatura de las hojas se relacionó directamente con la temperatura del suelo e inversamente con la humedad del suelo. Se comprobó que el tamaño del contenedor afecta las características físicas del sustrato y el crecimiento de las plantas, lo cual sugiere que con $0,02 \mathrm{~m}^{3}$ (con $45 \mathrm{~cm}$ altura) del contenedor las plantas pueden permanecer 180 días en vivero.

Palabras clave: frutales, volumen de sustrato, biomasa, temperatura, resistencia a la penetración. 


\begin{abstract}
Pot volume is a key factor for the propagation of cacao, since the pot size influences growth and development of seedlings which are crucial factors for crop establishment. In this work, the effect of different pot sizes 0.01 ; 0.02 y $0.1 \mathrm{~m}^{3}$ (substrate volume) and nursery duration on cocoa growth were compared, and changes in the substrate-confined were also evaluated. Seeds of cocoa were sowed in different pot sizes: 25,45 and $70 \mathrm{~cm}$. Leaf temperatures was measured and seedlings were collected to quantify partitioned biomass and taproot growth. Penetration resistance, temperature, humidity and porosity of the substrate were evaluated at the same time. The results evidenced significant differences $(\mathrm{P}<0.05)$ in total phytomass storage. In the larger containers $\left(0.1 \mathrm{~m}^{3}\right)$ there was higher total biomass, longer root length, $62.17 \mathrm{~cm}$ and the substrate had lower resistance to penetration $\left(0.52 \mathrm{Kg} \mathrm{cm}^{-2}\right)$ and temperature. Leaf temperature was directly related to the soil temperature and inversely related to the soil moisture. It was verified that the size of the container affects the physical characteristics of substrate and the plant growth, which suggests that with $0.02 \mathrm{~m}^{3}$ pot (of $45 \mathrm{~cm}$ height) the plants can last more than 180 days at the nursery.
\end{abstract}

Keywords: fruit, substrate volume, biomass, temperature, penetration resistance.

\section{INTRODUCCIÓN}

Las características del suelo se modifican periódicamente de forma natural pero pueden suceder cambios súbitos con el uso intensivo en actividades agropecuarias. El suelo confinado utilizado como sustrato, en vivero, acelera el proceso de cambio, como consecuencia se puede alterar el normal desarrollo de las plantas.

El cacao (Theobroma cacao L.) es un árbol originario de las selvas neotropicales, principalmente de la cuenca del Amazonas y posiblemente de la meseta Guyanesa (Lachenaud et al., 2007). Estudios más recientes describen la zona de origen en el triángulo amazónico entre Colombia. Ecuador y Perú (Motamayor et al., 2008); sin embargo, su zona de cultivo se extendió a través de la zona tropical y subtropical de Centro y Sur América, África occidental y Asia sudoriental, de tal manera que el rango de temperatura en el que se han desarrollado los cultivos, es mucho mayor que el de su hábitat natural (Daymond y Hadley, 2004).

En Colombia se ha priorizado el apoyo para el incremento del área de cultivos de cacao, que re- quiere de material vegetal con características de tamaño y desarrollo de la raíz que aseguren el establecimiento y longevidad del cultivo. Generalmente, este material de propagación proviene de viveros, donde las plántulas crecen en contenedores o bolsas con diversas condiciones de espacio y suelo para el desarrollo de sus raíces y que pueden afectar el crecimiento aéreo. El suelo depositado en contenedores puede modificar sus propiedades físicas y en consecuencia las plantas se pueden alterar, modificar o transformar. Los cambios en la densidad aparente del suelo pueden conducir a una disminución de poros y restricción en la penetración de las raíces (Lipiec y Stepniewski, 1995), como también disminuyen la disponibilidad de agua y la aireación, el crecimiento de la raíz (Gaitan et al., 2005) y la producción de las plantas (Domzal et al., 1991; Mapfumo et al., 1998; Arocena, 2000; Freddi, et al., 2006).

La zona donde el cacao crece espontáneamente se caracteriza por alta disponibilidad de agua con balance hídrico positivo. Pocas plantas cultivadas se muestran tan sensibles a la deficiencia de agua como esta especie y prácticamente todos sus procesos fisiológicos se ven afectados por la falta de humedad 
en el suelo (Amorim y Valle, 1992; GonzálesHuiman, 2008); por lo tanto, es transcendental una distribución apropiada de precipitaciones durante el año, en este sentido con un régimen pluvial, el suelo debe presentar drenaje perfecto (GonzálesHuiman, 2008).

Frimpong et al. (1996), trabajaron en condiciones de vivero con cacao amazónico y encontraron que las plántulas tolerantes al período de sequía alcanzaron mayor peso seco en las hojas y la raíz.

Varios factores ambientales controlan el crecimiento del cacao, entre ellos la precipitación, la temperatura y la humedad del suelo (Sena-Gomes y Kozlowsky, 1987), además, variables edáficas como resistencia a la penetración y porosidad del suelo influyen en el desarrollo de las plantas. Para obtener plántulas vigorosas se requiere conocer el tiempo de su permanencia en un determinado contenedor en condiciones de vivero. El objetivo de este trabajo fue comparar el efecto que tienen diferentes tamaños de contenedor en las propiedades físicas del suelo y la acumulación de fitomasa de plantas jóvenes de cacao.

\section{MATERIALES Y MÉTODOS}

El estudio se adelantó en casa de malla en la Universidad Nacional de Colombia Sede Medellín, ubicada a 6 6 15'46.89"LN, 7534'39.96 "LO y $1469 \mathrm{msnm}$. Durante el tiempo evaluado la evaporación promedio fue de $0,28 \mathrm{~mm}_{\text {día }}^{-1}$ y la temperatura máxima y mínima promedio fue de 35,0 y $17,0^{\circ} \mathrm{C}$, respectivamente.

La población experimental correspondió a 200 plantas obtenidas de semilla hibrida de cacao IMC-67, sembradas en contenedores cilíndricos plásticos de tres tamaños: pequeño de $0,01 \mathrm{~m}^{3}$, mediano de $0,02 \mathrm{~m}^{3} \mathrm{y}$ grande de $0,1 \mathrm{~m}^{3}$, con 25,45 y $70 \mathrm{~cm}$ de altura, en su orden. Se muestrearon tres plantas por cada tamaño de contenedor. Los muestreos se realizaron a los 240, 270 y 305 días después de sembradas (dds). Las variables medidas fueron: biomasa de la planta, y su distribución en hojas, tallo y raíz, longitud de raíz principal y temperatura de las hojas.

En el sustrato se evaluó la estabilidad de agregados del suelo con el diámetro medio ponderado en húmedo (DMPH) por el método de De Bood y De Lenner modificado por Ramírez (2008), densidad aparente por el método del cilindro biselado, densidad real por el método del picnómetro y resistencia a la penetración con el penetrómetro de bolsillo (Klutte, 1982). La temperatura del sustrato se tomó con un termómetro de punzón digital y la humedad por el método de humedad gravimétrica. El diseño experimental correspondió a Completamente al Azar (DCA). Se realizó un Análisis de Varianza y una prueba de comparación de medias de Duncan por medio del Software estadístico SAS ® versión 8.0, mediante el procedimiento GLM.

\section{RESULTADOS Y DISCUSIÓN}

Crecimiento de las plantas. Después de 305 días de emergencia, las plantas de cacao acumularon un $48 \%$ de biomasa en hojas, 33\% en tallo y la menor proporción, en la raíz (Tabla 1). La elongación de la raíz principal fue mayor en los contenedores grandes, con $62,7 \mathrm{~cm}$ de longitud. La raíz alcanzó la base del contenedor grande a los 270 días, la mediana a los 175 y la de contenedor pequeña a los 63 días.

Las variables medidas en la planta (Tabla 1) presentan diferencias significativas en la última evaluación, a los 305 días $(\mathrm{p}<0,05)$. La biomasa de tallo y de las hojas de las plantas en contenedor grande fue significativamente mayor que en los otros contenedores. Entre el mediano y el pequeño no se presentaron diferencias significativas. 
Tabla 1. Longitud de raíz pivotante, biomasa de raíz, biomasa aérea, biomasa total y temperatura de las hojas de cacao (T. cacao L.), en diferentes tamaños de contenedor.

\begin{tabular}{llllll}
\hline Tratamiento & $\begin{array}{c}\text { Longitud Raíz } \\
\text { principal } \\
\text { (cm) }\end{array}$ & $\begin{array}{l}\text { Biomasa } \\
\text { raíz (g) }\end{array}$ & $\begin{array}{c}\text { Biomasa } \\
\text { aérea (g) }\end{array}$ & $\begin{array}{c}\text { Biomasa } \\
\text { total (g) }\end{array}$ & $\begin{array}{c}\text { Temperatura } \\
\left.\text { hojas ( }{ }^{\circ} \mathbf{C}\right)\end{array}$ \\
\hline MG240 & $31,17 \mathrm{c} \mathrm{d}$ & $0,93 \mathrm{c} \mathrm{d}$ & $3,47 \mathrm{~b}$ & $4,40 \mathrm{~b}$ & $16,27 \mathrm{e}$ \\
MG270 & $62,17 \mathrm{a}$ & $1,33 \mathrm{c} \mathrm{d}$ & $6,67 \mathrm{~b}$ & $8,00 \mathrm{~b}$ & $24,67 \mathrm{a} \mathrm{b}$ \\
MG305 & $50,00 \mathrm{a} \mathrm{b}$ & $3,97 \mathrm{a}$ & $17,00 \mathrm{a}$ & $20,97 \mathrm{a}$ & $24,87 \mathrm{a}$ \\
MM240 & $35,67 \mathrm{~b} \mathrm{c} \mathrm{d}$ & $0,40 \mathrm{~d}$ & $1,76 \mathrm{~b}$ & $2,17 \mathrm{~b}$ & $20,03 \mathrm{~d}$ \\
MM270 & $43,17 \mathrm{~b} \mathrm{c}$ & $2,00 \mathrm{~b} \mathrm{c}$ & $4,50 \mathrm{~b}$ & $6,50 \mathrm{~b}$ & $22,63 \mathrm{c}$ \\
MM305 & $43,50 \mathrm{~b} \mathrm{c}$ & $2,80 \mathrm{~b}$ & $6,33 \mathrm{~b}$ & $9,13 \mathrm{~b}$ & $22,63 \mathrm{c}$ \\
MP240 & $24,17 \mathrm{~d}$ & $0,60 \mathrm{~d}$ & $2,94 \mathrm{~b}$ & $3,53 \mathrm{~b}$ & $23,47 \mathrm{a} \mathrm{b} \mathrm{c}$ \\
MP270 & $28,00 \mathrm{c}$ & $0,60 \mathrm{~d}$ & $2,36 \mathrm{~b}$ & $2,97 \mathrm{~b}$ & $23,23 \mathrm{a} \mathrm{b} \mathrm{c}$ \\
MP305 & $21,67 \mathrm{~d}$ & $1,20 \mathrm{c} \mathrm{d}$ & $3,17 \mathrm{~b}$ & $4,37 \mathrm{~b}$ & $22,83 \mathrm{~b} \mathrm{c}$ \\
\hline
\end{tabular}

${ }^{*}$ MG: contenedor grande $\left(0,1 \mathrm{~m}^{3}\right)$, MM: contenedor mediano (0,02 m3), MP: contenedor pequeño $\left(0,01 \mathrm{~m}^{3}\right), 240,270$ y 305 días después de la siembra. Las medias con diferente letra, estadísticamente presentan diferencias significativas $($ Duncan $P=0,05)$.

La biomasa y la longitud de la raíz principal evidenciaron cambios con relación al volumen de los contenedores durante el periodo evaluado. Las plantas de mayor crecimiento fueron las sembradas en contenedores grandes. La disminución de tamaño fue significativa y proporcional al volumen del contenedor. Se puede inferir que el volumen y la altura del contenedor condicionaron el crecimiento de la raíz y este influyó sobre la acumulación de biomasa de la parte aérea.

De forma similar, estudios realizados por Gutiérrez et al (2011) en Cacao, Ouma (2007) en Mango, y Arizaleta y Pire (2008) en Cafeto, señalan que en las bolsas de menor tamaño hubo menor magnitud de todas las mediciones en la planta y que en los recipientes de mayor tamaño, hubo mayor acumulación de biomasa en la raíz. En este sentido, Pritchett (1986) señala que el volumen de suelo disponible para las raíces, que está determinado por la profundidad, simulada con la altura del contenedor, es uno de los principales factores que influye en el crecimiento de los árboles. Alvarenga y Cruz (2003) anotan que la habilidad de las plantas para explorar el suelo depende en gran parte de la distribución de las raíces en el perfil y de las características del suelo (Henríquez y Cabalceta, 1999; Porta et al., 2003, Ramírez, 2016).

La mayor biomasa total se obtuvo en las plantas establecidas en contenedores de mayor volumen, a los 305 días, con peso promedio de 20,97g (Tabla. 1) y la menor biomasa en las plantas de contenedor pequeño. Este crecimiento diferencial se puede atribuir al mayor y significativo desarrollo de la raíz, dado el amplio volumen de exploración y la baja impedancia del suelo en los contenedores grandes que influyó favorable y significativamente sobre el crecimiento aéreo (Ramírez y Salazar, 2006; Bengough et al., 2011).

La relación proporcional entre la tasa de crecimiento de las raíces respecto a la parte aérea corresponde a un crecimiento equilibrado (Fageria 
et al, 1997; Mccarthy y Enquist, 2007; Kobe et al, 2010) como sucedió en los contenedores grandes, donde se conservó proporcional cuando no hubo limitantes. En el caso de restricciones en el suelo, como lo es menor volumen, la demanda de la raíz es mayor (Kobe et al., 2010) y la relación disminuyó, es decir la proporción de crecimiento radical se incrementó como ocurrió en los contenedores medianos, después de 270 días.

En esta investigación, se evidenció que la planta mantiene un balance en la distribución de fotoasimilados sin alteración proporcional entre la biomasa aérea y la radical (Figura 1), sólo modifica la cantidad de biomasa acumulada dependiendo del volumen de suelo disponible en el contenedor. En este sentido, Haolin et al. (2008) indicaron que la repartición de fotoasimilados se puede alterar por distintos factores, así mismo Mokany et al. (2006) encontraron que la relación de biomasa radical y aérea se afecta significativamente con los factores climáticos y del suelo.

La distribución de biomasa está influenciada por diversos factores que al dilucidarlos y conocer su efecto regulatorio permitirá mayores aproxima- ciones para la estimación de la acumulación de carbono terrestre (Cairns et al., 1997; Mokany et al., 2005) y del crecimiento de los cultivos; el volumen de sustrato de los contenedores utilizados es un factor que simula la profundidad disponible en el suelo para el crecimiento de las plantas. En este sentido, las mediciones realizadas sugieren que con diez meses de crecimiento en un suelo de 25 o de $45 \mathrm{~cm}$ de profundidad, las plantas de cacao solo alcanzan el 21 o el 50\% de la biomasa, respectivamente, en relación con las que crecen en un suelo con $70 \mathrm{~cm}$ de profundidad.

Sustrato. La menor resistencia a la penetración se presentó en los contenedores grandes con un valor de $0,52 \mathrm{~kg} \mathrm{~cm}^{-2}$ alos 240 días (Tabla 2). Entrelos otros tratamientos no hubo diferencias significativas, la impedancia está el rango de valores (Ramírez y Salazar, 2006, Ramírez, 2016) que permiten el desarrollo de las raíces sin dificultad. El diámetro medio ponderado en húmedo (DMPH) no presentó diferencias entre los tamaños de contenedor y fue cercano a $2 \mathrm{~mm}$ en todos los casos, condición ideal para el desarrollo de las raíces debido a que genera la porosidad que permite suficiente retención de agua y aireación apropiada (Ramírez, 2016).

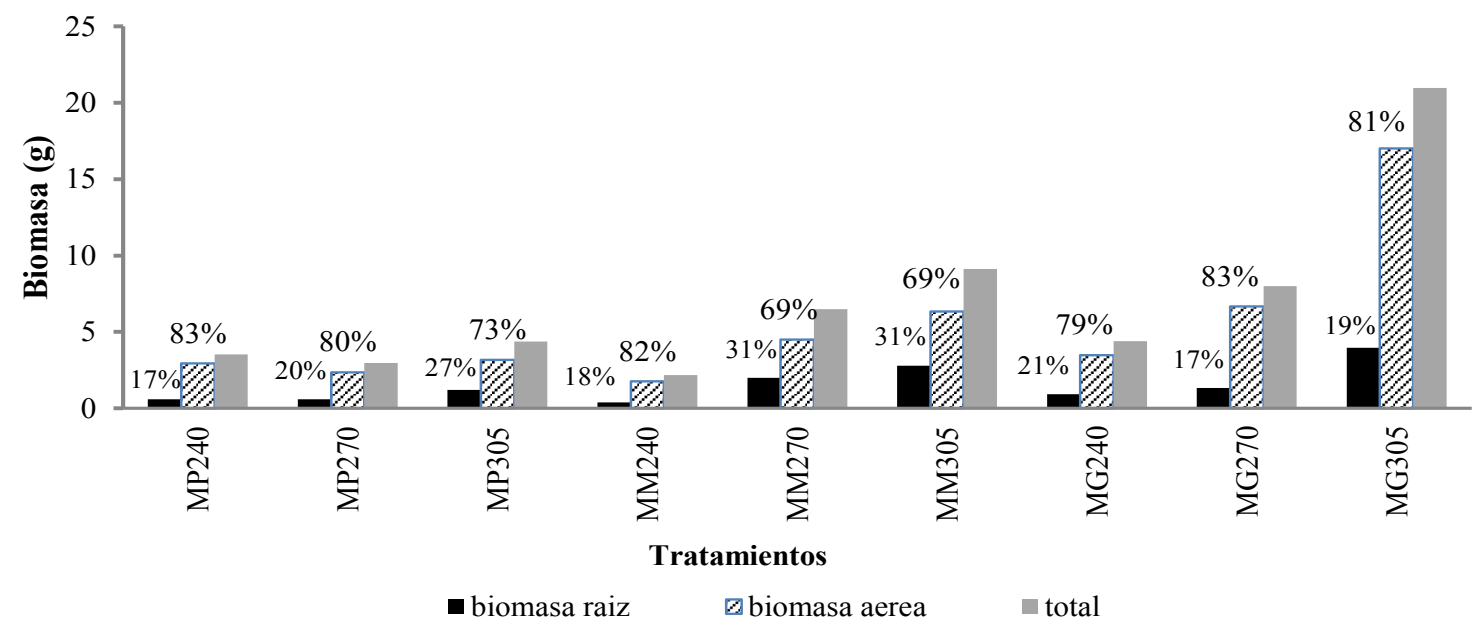

Figura 1. Biomasa total y proporción de biomasa radical y biomasa aérea de plantas de cacao. MP:contenedor pequeño $(0,01 \mathrm{~m} 3)$. MM:contenedor mediano $(0,02 \mathrm{~m} 3)$ MG: contenedor grande $\left(0,1 \mathrm{~m}^{3}\right), 240,270$ y 305 días después de la siembra. 
En cuanto a la porosidad, en los contenedores pequeños se presentó la mayor cantidad de poros sin diferencias significativas entre los períodos evaluados. La porosidad regula el almacenamiento y movimiento de gases y agua e influye en el desarrollo de las plantas, debido a que facilitan la penetración por parte de las raíces (Guidi et al., 1985). Bengough et al. (2011) señalan que la resistencia a la penetración mayor a $2 \mathrm{MPa}\left(20,4 \mathrm{~kg} \mathrm{~cm}^{-2}\right)$ y la porosidad menor al $10 \%$ se consideran límites críticos para la elongación de las raíces. Con base en estas referencias, todos los contenedores presentaron alta porosidad (mayor a 69\%) y baja impedancia (menor de $1 \mathrm{Kg} \mathrm{cm}^{-2}$ ) clasificadas en el rango de condiciones óptimas (Taylor, 1980; Ramírez y Salazar, 2006; Ramírez, 2016).

Los cambios en la humedad ocasionaron cambios en la temperatura del suelo. A mayor humedad, menor temperatura. Las propiedades físicas del suelo son interdependientes. La ocurrencia de cambios en una de ellas, normalmente, modifica otras (Zanette et al., 2007; Ramírez, 2008). Se observó que la humedad del suelo tiene relación directa con el tamaño de contenedor durante el tiempo evaluado, encontrando un mayor contenido de humedad en los contenedores grandes y menor en los pequeños (Tabla 2). La humedad y la temperatura del suelo tienen efecto directo sobre el crecimiento de las plantas, así como en los procesos químicos y el grado de actividad de diversos tipos de organismos en el suelo (Gavye,1979; Henríquez y Cabalceta, 1999; Porta et al., 2003) que influyen en el crecimiento.

El crecimiento de la raíz se relaciona inversamente con la resistencia a la penetración del suelo (Figura 2) que coincide con lo reportado por Freddi et al. (2006). Igualmente, la compactación del suelo puede reducir la penetración de raíces (Bengough et al., 2001) y alterar el equilibrio de la proporción de gases del suelo y la disponibilidad de agua y nutrientes para las plantas (Ribeiro, 1995; Stirzaker et al., 1996; Grohmann y Queiroz, 1966). En este sentido, los contenedores pequeños presentaron la mayor resistencia a la penetración, 0.97$0,98 \mathrm{Kg} \mathrm{cm}^{-2}$, condición que afectó el desarrollo de la raíz y en consecuencia se disminuyó el crecimiento de las plantas (Figura 1).

Tabla 2. Resultados de humedad y temperatura del suelo, porosidad, resistencia a la penetración y diámetro medio ponderado de los agregados (DMPH), en diferentes tamaños de contenedor.

\begin{tabular}{llllll}
\hline Tratamiento & $\begin{array}{c}\text { Humedad del } \\
\text { Suelo (\%) }\end{array}$ & $\begin{array}{c}\text { Temperatura del } \\
\text { Suelo }\left({ }^{\circ} \mathbf{C}\right)\end{array}$ & $\begin{array}{c}\text { Porosidad } \\
\mathbf{( \% )}\end{array}$ & $\begin{array}{c}\text { Resistencia a la } \\
\text { Penetración }\left(\mathbf{K g ~ c m}^{-2}\right)\end{array}$ & DPMH (mm) \\
\hline MG 240 & $77,59 \mathrm{a}$ & $15,40 \mathrm{~d}$ & $69,60 \mathrm{c}$ & $0,52 \mathrm{c}$ & $2,10 \mathrm{a}$ \\
MG 270 & $44,24 \mathrm{~b}$ & $22,90 \mathrm{a} \mathrm{b}$ & $69,85 \mathrm{c}$ & $0,77 \mathrm{~b}$ & $2,20 \mathrm{a}$ \\
MG 305 & $43,89 \mathrm{~b}$ & $22,83 \mathrm{a} \mathrm{b}$ & $70,56 \mathrm{c}$ & $1,14 \mathrm{ab}$ & $2,40 \mathrm{a}$ \\
MM 240 & $68,97 \mathrm{a}$ & $20,63 \mathrm{c}$ & $73,76 \mathrm{~b} \mathrm{c}$ & $0,84 \mathrm{ab}$ & $2,13 \mathrm{a}$ \\
MM 270 & $45,33 \mathrm{~b}$ & $21,83 \mathrm{~b} \mathrm{c}$ & $71,39 \mathrm{~b}$ & $0,93 \mathrm{a} \mathrm{b}$ & $2,03 \mathrm{a}$ \\
MM 305 & $37,60 \mathrm{~b} \mathrm{c}$ & $23,83 \mathrm{a}$ & $78,07 \mathrm{a}$ & $1,07 \mathrm{a} \mathrm{b}$ & $2,13 \mathrm{a}$ \\
MP 240 & $41,45 \mathrm{~b} \mathrm{c}$ & $21,63 \mathrm{~b} \mathrm{c}$ & $78,20 \mathrm{a}$ & $0,98 \mathrm{a} \mathrm{b}$ & $2,20 \mathrm{a}$ \\
MP 270 & $31,08 \mathrm{~b} \mathrm{c}$ & $24,07 \mathrm{a}$ & $77,68 \mathrm{a}$ & $0,97 \mathrm{a} \mathrm{b}$ & $2,20 \mathrm{a}$ \\
MP 305 & $27,39 \mathrm{c}$ & $22,23 \mathrm{~b}$ & $77,95 \mathrm{a}$ & $0,90 \mathrm{a} \mathrm{b}$ & $2,23 \mathrm{a}$ \\
\hline
\end{tabular}

*MG: Contenedor grande $\left(0,1 \mathrm{~m}^{3}\right)$, MM: Contenedor mediano $\left(0,02 \mathrm{~m}^{3}\right)$, MP: Contenedor pequeño $\left(0,01 \mathrm{~m}^{3}\right) .240,270$ y 305 días después de la siembra. Las medias con diferente letra, estadísticamente presentan diferencias significativas, (Duncan $\mathrm{P}=0,05)$. 




Figura 2. Relación de longitud de la raíz pivotante y resistencia a la penetración con diferentes contenedores y tiempos.

Temperatura de las hojas y del suelo. La temperatura del suelo y la de las hojas tienen una relación directa. A medida que aumenta la temperatura del suelo, también se incrementa la temperatura de las hojas (Figura 3). Por el contrario existe una relación inversa entre la humedad y la temperatura del suelo, porque a medida que la humedad disminuye, la temperatura aumenta. Aunque la temperatura ambiente presentó un gradiente bas- tante amplio, la planta reguló la temperatura de las hojas a $25^{\circ} \mathrm{C}$, indicando con esto su capacidad de estabilizar la temperatura a las condiciones apropiadas para su funcionamiento y desarrollo (Sena-Gomes y Kozlowsky, 1987) cuando el suelo presenta humedad disponible fácilmente, con potencial mátrico cercano a $0,03 \mathrm{MPa}$. En este caso permaneció con una humedad volumétrica próxima al 30\%.

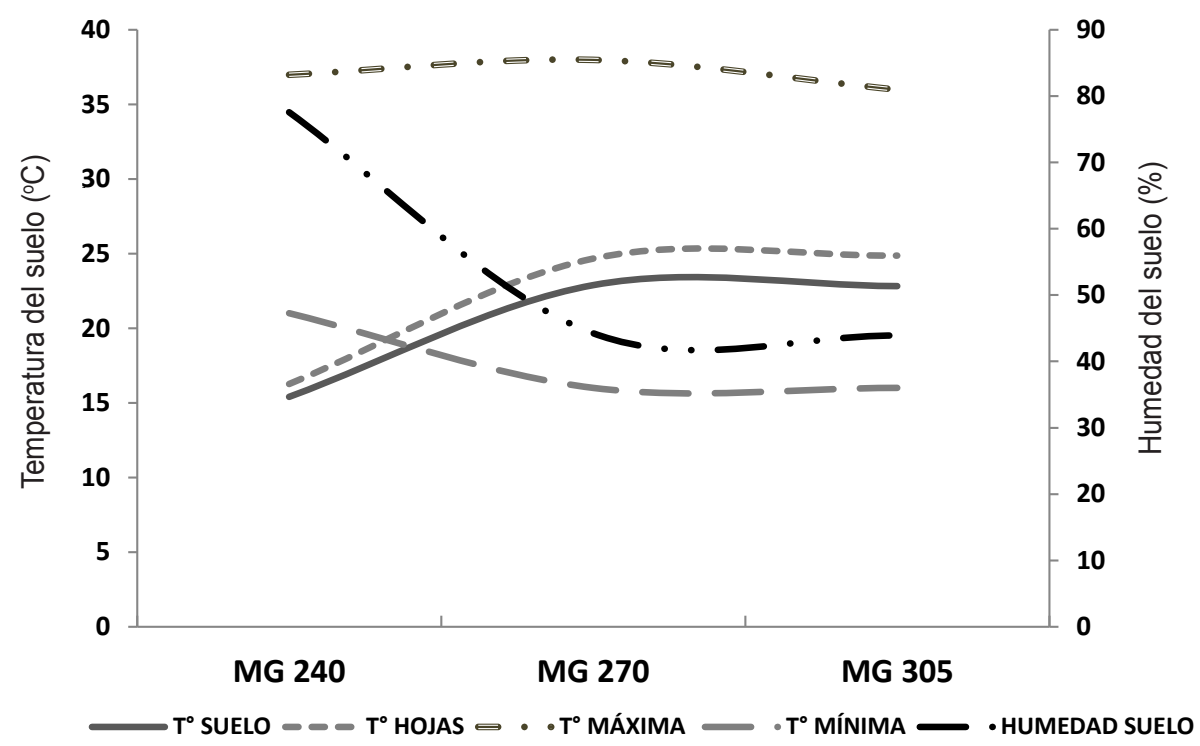

Figura 3. Relación temperatura del suelo, temperatura de las hojas, temperatura ambiente y humedad del suelo en los contenedores de mayor tamaño a lo largo del tiempo de evaluación. 


\section{CONCLUSIONES}

El crecimiento longitudinal de la raíz pivotante se detiene cuando la resistencia a la penetración en el suelo es superior a $0,7 \mathrm{~kg} \mathrm{~cm}^{-2}$ y afecta la acumulación de biomasa aérea, disminuyendo el vigor de las plántulas.

La temperatura de las hojas está relacionada inversamente con la humedad del suelo y directamente con la temperatura del suelo. Las hojas regularon su temperatura en $25^{\circ} \mathrm{C}$, con un límite mínimo de humedad del suelo de $27 \%$.

El tiempo de permanencia de las plantas de Cacao en vivero, sin afectar el desarrollo de la raíz, depende de la profundidad del contenedor, con 25 y $45 \mathrm{~cm}$, se puede mantener 60 y 180 días respectivamente, antes del trasplante a sitio definitivo.

La porosidad, la resistencia a la penetración y la humedad del sustrato en los contenedores pequeños, $0,01 \mathrm{~m} 3$, no presentaron cambios en el período evaluado, 305 días. En los contenedores grandes se incrementó significativamente la resistencia a la penetración y presentó la menor porosidad. A mayor tamaño de contenedor, los cambios en el sustrato son de mayor magnitud.

\section{REFERENCIAS BIBLIOGRÁFICAS}

1. Alvarenga, R.; Cruz, J. 2003. Manejo de solos e agricultura irrigada. pp. 70-106. In: Resende, M., P. Alburquerque y L. Couto. A cultura do milho irrigado. Embrapa Informação Tecnológica. Brasilia, Brasil.

2. Amorim, S.; Valle. R. 1992. Efeito da temperatura radicular na resistência ao movimiento de água no cacaueiro (Theobroma cacao L.). Acta Botanica Brasilera. 6(1):55 - 64. doi:10.1590/ S0102-33061992000100004.
3. Arocena, J.M. 2000. Cations in solution from forest soils subjected to forest floor removal and compaction treatments. Forest Ecology y Managament 133:71 - 80. doi: 10.1016/S03781127(99)00299-6

4. Arizaleta, M.; Pire, R. 2008. Respuesta de plántulas de cafeto al tamaño de la bolsa y fertilización con nitrógeno y fósforo en vivero. Agrociencia 42:47 - 55. doi: 10.1097/00010694197508000-00015.

5. Bengough, A. G.; Campbell, D.; 0’ Sullivan, M. 2001. Penetrometer techniques in relation to soil compaction y root growth. pp. 377 - 403. En: Smith, K. A.; Mullins, C. E. Soil y environmental analysis: physical methods. Second edition. Edinburgh: Marcel Dekker.

6. Bengough A.G.; Mckenzie, B.M.; Hallett P.D.; Valentine, T.A. 2011. Root elongation, water stress, and mechanical impedance: a review of limiting stresses and beneficial root tip traits. Journal of Experimental Botany. 62(1):59 - 68. doi: $10.1093 / j x b / e r q 350$

7. Cairns, M.; Brown, S.; Helmer, E.; Baumgardner, G. 1997. Root biomass allocation in the world's upland forests. Oecologia. 111(1):1 - 11. doi: $10.1007 /$ S004420050201.

8. Daymond, A.; Hadley, P. 2004. The effects of temperature and light integral on early vegetative growth y chlorophyll fluorescence of four contrasting genotypes of cacao (Theobroma cacao). Annals of Applied Biology. 145:257 - 262. doi: 10.1111/j.1744-7348.2004.tb00381.x

9. Domzal, H.; Glinski, J.; Lipiec, I.1991. Soil compaction research in Poly. pp. 99 - 109. En: Van Ouwerkerk, C. (Ed.), Soil Compaction and Plant Productivity. Elsevier, Amsterdam, the Netherlys.

10. Fageria, N.; Baligar, V.; Wright, R.J. 1997. Soil environment and root growth dynamics of field crops. Recent. Res. Devel. Agron. 1:15 - 28.

11. Freddi, 0., Morel, P.; Valdenir-Veronesi, J.:.Carvalho, G.J. 2006. Produtividade do milho 
relacionada com a resistência mecânica à penetração do solo sob preparo convencional. Engenharia Agrícola. Jaboticabal. 26(1):113 - 121. doi: 10.1590/S0100-69162006000100013.

12. Frimpong, E.; Adu-Ampomah, Y.; Karimu, A. 1996. Efforts to breed for drought resistant cacao in Ghana. pp. 24 - 25. Proceedings of the $12^{\text {th }}$ International Cacao Research Conference, Bahía, Brasil.

13. Gaitan, J.; Penón, E.; Costa, M. 2005. Distribución de raíces finas de eucalyptus globulus ssp. Maidenii y su relación con algunas propiedades del suelo. Ciência Florestal, Santa Maria, 15(1):33 - 41. doi: 10.5902/198050981822.

14. Grohmann. F. y J.P. Queiroz. 1996. Efeito da compactação artificial de dois solos limoargiloso sobre a penetração de raízes de arroz. Bragantia, Campinas. 25(39):421 - 31.

15. Gonzáles-Huiman, F. 2008. Ecofisiologia del Cacao. Tingo Maria - Peru: Diplomado u.n.a.s. In: http://diplomado2007unas.blogspot.com. co/2008/01/ecofisiologia-del-cacao.html; consulted: march, 2016.

16. Guidi, G.; Poggio G.; Petruzzell. G. 1985. The porosity of soil aggregates from bulk soil and from soil adhering to roots. Plant y Soil. 87:311 $-314$.

17. Gutiérrez, M.; Gómez, R.; Rodríguez, N.F. 2011. Comportamiento del crecimiento de plántulas de cacao (Theobroma cacao L.), en vivero, sembradas en diferentes volúmenes de sustrato. Corpoica Ciencia y Tecnología Agropecuaria. 12(1):33 - 42.

18. Haolin, G.; Wang, Y.; Wang, F.; Jia. B. 2008. Dynamics of root-shoot ratio and environmental effective factors of recovering Leymus chinensis steppe vegetation in Inner Mongolia, China. Acta Ecológica Sínica. 28(10):4629 - 4634.

19. Henríquez, C.; Cabalceta. G. 1999. Guía práctica para el estudio introductorio de los suelos con un enfoque Agrícola. ACCS. primera edición. San José, Costa Rica. 111p.
20. Kobe, R.K.; Iyer, M.; Walters, M.B. 2010. Optimal partitioning theory revisited: Nonstructural carbohydrates dominate root mass responses to nitrogen. Ecology. 91:166 - 179.

21. Klutte, A. 1982. Retention laboratory methods. Physical and mineralogical methods. Second edition by klutte. 235 - 240p.

22. Lachenaud, P.; Paulin, D.; Ducamp, M.; Thevenin. J. 2007. Twenty years of agronomic evaluation of wild cocoa trees (Theobroma cacao L.) from French Guiana. Scientia Horticulturae. 113(4):313 - 321.

23. Lipiec, J.; Stepniewski. W.1995. Effects of soil compaction and tillage systems on uptake and losses of nutrients. Soil Tillage Research. 35:37 - 52. doi:10.1016/0167-1987(95)00474-7.

24. Mccarthy, M.; Enquist. B. 2007. Consistency between an allometric approach and optimal partitioning theory in global patterns of plant biomass allocation. Funct. Ecol. 21:713 - 720.

25. Mapfumo, E., D.S. Chanasyk. M.A. Naeth Y V.S. Baron. 1998. Forage growth and yield components as influenced by subsurface compaction. Agronomy Journal. 90:805 - 812. doi:10.2134/ agronj1998.00021962009000060015x.

26. Mokany, K.; Raison, J.;Prokushkin. A. 2005. Critical analysis of root: shoot ratios in terrestrial biomes. Global Change Biology. 11:1 - 3. doi: 10.1111/j.1365-2486.2005.001043.x.

27. Mokany, K.; Raison, R.;Prokushkin. A. 2006. Critical analysis of root: shoot ratios in terrestrial biomes. Global Change Biology. 12:84 - 96. doi: 10.1111/j.1365-2486.2005.001043.x.

28. Motamayor, J.; Lachenaud, P.; Da Silva E Mota, J.; Loor, R.; Kuhn, D.N. J.; Brown, S.; Schnel, R. 2008. Geographic and Genetic Population Differentiation of the Amazonian Chocolate Tree (Theobroma cacao L).3(10):1 - 8. doi: 10.1371/ journal.pone.0003311. 
29. Ouma G. 2007. Effect of different container sizes and irrigation frequency on the morphological and physiological characteristics of mango (Mangifera indica) rootstock seedlings. International Journal of Botany. 3:260 - 268. doi: 10.3923/ijb.2007.260.268.

30. Porta, C.J.; López-Acevedo, R.M.; Roquero, D.C. 2003. Edafología para la agricultura y el medio ambiente. Ediciones Mundi Prensa. España. Tercera edición. 929p.

31. Pritchett, W.L. 1986. Los suelos forestales: propiedades, conservación y mejoramiento. Noriega, Editorial Limusa, 643 p.

32. Ramírez, R.; Salazar, C. 2006. Cambios de la resistencia a la penetración con diferentes sistemas de manejo y su relación con algunas propiedades físicas en un Yisol del municipio de - Marinilla (Antioquia). P. XX En: XIII Congreso Colombiano de la ciencia del suelo. 4-6 de octubre. Bogotá, Colombia. Sociedad colombiana de la Ciencia del Suelo.

33. Ramírez, R. 2008. Cambios en los macroagregados por prácticas agrícolas y procesos de recuperación en un Andisol, en la Vereda Montañitas, Municipio De Marinilla, Oriente Antioqueno Colombiano. Tesis Doctoral. Universidad Nacional de Colombia. Palmira, Colombia. 167p.

34. Ramírez, R. 2016. Fertilidad integral del suelo. pp. 41 - 56. En: Actualización en fertilidad del suelo. Armando Torrente editor. Grafiweb impresores publicistas. Bogotá Colombia. 233p.

35. Ribeiro Jr., P. 1995. Métodos geoestatísticos no estudo da variabilidade espacial de parâmetros do solo. Piracicaba: ESALQ. 99p.

36. Sena-Gomes, A. R. Y T.T. Kozlowsky. 1987. Effects of temperature on growth and water relations of cacao (Theobroma cacao var. Comum) seedlings. Plant and Soil 103:3 - 11. doi: 10.1007/BF02370661.

37. Stirzaker, R.; Passioura, J.; Wilms. Y. 1996. Soil structure and plant growth: impact of bulk density and biopores. Plant and Soil, Dordrecht. 185(1):151 - 162. doi: 10.1007/BF02257571.
38. Taylor H.M. 1980. Mechanical impedance to root growth. pp 389 - 404. En: Soil Related Constraints to Food Production in the Tropics. The International Rice Research Institute And The New York State College Of Agriculture And Life Sciences, Cornell University In Cooperation With The University Consortium Of Soils For The Tropics. 478p

39. Zanette. S.; Sampaio, C.; Silvestre, M.; Boas, M.; M. Uribe-Opazo M. Queiroz. 2007. Análise espacial da umidade do solo cultivado com soja sob dois sistemas de manejo. Revista Brasileira de Engenharia Agrícola e Ambiental. 11(3):239 - 247. doi: 10.1590/S141543662007000300001. 